\title{
Infección por Sphingobacterium multivorum en un niño gran quemado
}

\author{
Sphingobacterium multivorum infection in a child with an extensive burn
}

\author{
Esmeralda Highton', Jorge F. Guarracino², Máximo Navarro², José L. Pinheiro ${ }^{3}$, Adela Isasmendi y M. Teresa Rosanova ${ }^{1}$
}

'Servicio de Control Epidemiológico e Infectología. Hospital Profesor Dr. Juan P. Garrahan, Buenos Aires, Argentina.

${ }^{2}$ Cirugía Plástica y Quemados. Hospital Profesor Dr. Juan P. Garrahan, Buenos Aires, Argentina.

${ }^{3}$ Servicio de Microbiología, Hospital Profesor Dr. Juan P. Garrahan, Buenos Aires, Argentina.

Recibido: 6 de enero de 2021 / Aceptado: 13 de abril de 2021

\section{Resumen}

Sphingobacterium multivorum es un bacilo gramnegativo no fermentador, que infrecuentemente causa enfermedad en humanos. En la literatura médica existen escasos reportes de infecciones causadas por este microorganismo, en general, en hospederos con alguna comorbilidad. Presentamos el primer caso reportado, según nuestro conocimiento, de una infección de piel y tejidos blandos por Sphingobacterium multivorum en un paciente pediátrico gran quemado.

Palabras clave: Sphingobacterium multivorum; infección intrahospitalaria; quemados; niños.

\section{Introducción}

S phingobacterium multivorum es un bacilo gramnegativo, no fermentador, que infrecuentemente causa enfermedad en huma$\operatorname{nos}^{1}$.

El género Sphingobacterium engloba a microorganismos que antes se clasificaban como especies de Flavobacterium. El género Sphyngobacterium incluye varias especies: $S$. anhuiense, $S$. antarcticus, $S$. bambusae, S. canadense, $S$. composti, S. daejeonense, S. faecium, $S$. heparinum, S. kitahiroshimense, S. multivorum, S. piscium, S. shayense, $S$. siyangense, $S$. spiritivorum y $S$. thalpophilum ${ }^{2}$. Casi todas las cepas aisladas en seres humanos son $S$. multivorum o $S$. spiritivorum.

En la literatura médica existen escasos reportes de infecciones causadas por este microorganismo, en general, en hospederos con alguna comorbilidad. Sin embargo, en los últimos años se han descrito casos de infección en individuos inmunocompetentes $\mathrm{s}^{3-4}$.

Presentamos el caso clínico de un paciente pediátrico gran quemado que en relación a signos clínicos de sepsis, se confirmó una infección de piel y tejidos blandos por $S$. multivorum.

\section{Abstract}

Sphingobacterium multivorum is a non-fermentative gram-negative bacillus that rarely causes human infections. In the medical literature, the few reported cases of infections resulting from $S$. multivorum usually occurred in patients with an associated comorbidity. We present the first case report, according our knowledge, of a skin and soft tissue infection by $S$. multivorum infection in a pediatric patient after a burn injury.

Keywords: Sphingobacterium multivorum; nosocomial infection; burn; children.

\section{Caso clínico}

Varón de 6 años de edad, previamente sano, que sufrió una quemadura extensa por fuego directo con inhalación de humo causado por un incendio doméstico. Fue derivado a un centro terciario con quemaduras en cara y vibrisas, donde se realizó intubación orotraqueal por sospecha de un síndrome inhalatorio.

$\mathrm{Al}$ ingreso se evidenció compromiso de rostro, cuello, tórax, abdomen, dorso, extremidades superiores e inferiores; confirmándose una quemadura del $60 \%$ tipo A-AB. Se encontraba en asistencia respiratoria mecánica. Se instaló un catéter venoso central y se inició reposición de volumen. Por inestabilidad hemodinámica requirió apoyo con aminas vasoactivas (adrenalina y noradrenalina). Se realizaron escarectomías seriadas en el pabellón quirúrgico, con aplicación de sulfadiazina de plata tópica y cubriéndose las heridas con vendaje compresivo posterior a dichos procedimientos.

A las tres semanas de internación tuvo un episodio de descompensación hemodinámica, posterior a un procedimiento quirúrgico de escarectomía y autoinjerto. El hemograma mostró una leucocitosis de 
29.000 céls $/ \mathrm{mm}^{3}$, de predominio neutrófilos (91\%), una anemia con hemoglobina $7,5 \mathrm{~g} / \mathrm{dl}$ y hematocrito $22 \%$ y plaquetas normales. La proteína C reactiva se elevó a 126 $\mathrm{mg} / \mathrm{l}$. Requirió expansión de la volemia con coloides, aumento de la dosis de fármacos inotrópicos y transfusión de glóbulos rojos.

El cultivo de tejido de la biopsia del brazo derecho, tomado en el último procedimiento quirúrgico ante la presencia de secreción purulenta, fue positivo para Sphingobacterium multivorum.

El aislado fue sensible a meropenem, cotrimoxazol y ciprofloxacina. La identificación bacteriana se realizó por técnica de MALDI-TOF MS (Vitek-MS, bioMérieux). Las pruebas de sensibilidad in vitro a los distintos antimicrobianos se realizaron por métodos automatizados (Vitek $2 \mathrm{C}$, bioMérieux) y por el método de difusión con discos, según CLSI. Los hemocultivos resultaron negativos.

Debido al deterioro clínico y parámetros de laboratorio inflamatorios había iniciado tratamiento empírico con vancomicina y colistín, considerando la epidemiología de la unidad de cuidados intensivos local. Con el resultado del cultivo de tejido se cambió la terapia antibacteriana a meropenem.

En el estudio histopatológico se informó, a la microscopía, tejido fibroadiposo con depósitos de fibrina y moderado infiltrado inflamatorio mixto, evidenciando signos indirectos de infección. Se logró descenso de los fármacos inotrópicos, hasta la suspensión de los mismos a las $48 \mathrm{~h}$ del ajuste del tratamiento antibacteriano.

El paciente sobrevivió sin presentar nuevas infecciones, quedando con secuelas permanentes como cicatrices cutáneas y deformidad en las extremidades superiores.

\section{Discusión}

Las especies del género Sphingobacterium contienen grandes cantidades de esfingofosfolípidos en sus membranas celulares, además de poseer otras características taxonómicas que las distinguen de las flavobacterias.

Sphingobacterium son bacilos rectos gramnegativos, aerobios estrictos, que se desarrollan con facilidad en los medios de cultivos utilizados en la práctica diaria. Las pruebas de ADNasa, oxidasa, catalasa y ureasa son positivas e indol negativas. Producen colonias de color amarillo claro. La identificación puede realizarse mediante la técnica de espectrometría de masas (MALDI-TOF MS Vitek-MS, bioMérieux), sistemas automatizados y semiautomatizados (Vitek 2C y API 20NE bioMérieux). En general, las especies de Sphingobacterium son resistentes a aminoglucósidos y polimixina $\mathrm{B}^{2-6}$. S. multivorum puede producir $\beta$-lactamasas de espectro extendido y metalo- $\beta$ lactamasas, confiriéndole resistencia a las cefalosporinas de tercera generación y carbapenémicos, respectivamen$t^{7}$. En general, es sensible a cotrimoxazol, quinolonas y tetraciclinas. La correcta identificación y determinación de la susceptibilidad antimicrobana es muy importante para un tratamiento adecuado.

Sphingobacterium multivorum es una bacteria ubicua en la naturaleza, saprófita, raramente involucrada en infecciones en humanos ${ }^{1-8}$. Se ha aislado en el suelo, plantas, alimentos y fuentes de agua ${ }^{7}$. Es capaz de contaminar medios de cultivo de laboratorio y crecer en antisépticos y desinfectantes.

Previamente, no se conocía su rol patógeno en humanos; sin embargo, en los últimos años cada vez se han descrito con mayor frecuencia casos de infección por $S$. multivorum alrededor del mundo.

En general, los casos de infecciones por S. multivorum ocurren en pacientes con alguna comorbilidad, como diabetes mellitus, enfermedad renal crónica terminal, en hemodiálisis, pacientes oncológicos en tratamiento quimioterápico, con cirrosis, VIH o fibrosis quística ${ }^{9-11}$. La mayoría de las infecciones son de adquisición intrahospitalaria, sin embargo, también se han reportado casos provenientes de la comunidad ${ }^{9}$.

Dentro de las formas de presentación clínica reportadas se encuentran: un caso de fascitis necrosante en un paciente con diabetes mellitus, un shock séptico en un paciente con una artritis reumatoidea en tratamiento con corticoesteroides, cuadros de sepsis y bacteriemia en hospederos inmunocomprometidos y en hemodiálisis, peritonitis, celulitis, endocarditis, meningitis, artritis séptica en paciente sometidos a trasplante hepático e infección crónica del tracto respiratorio en pacientes con fibrosis quística, entre otros ${ }^{7,12-15}$.

Recientemente se publicó el caso de un adulto joven de 28 años inmunocompetente con una bacteriemia y meningitis aguda de la comunidad por $S$. mutivorum luego de un trauma cutáneo y un caso de shock séptico secundario a una bacteriemia en una mujer inmunocompetente de 67 años de edad ${ }^{14}$.

A pesar de una búsqueda detallada en la literatura especializada, no encontramos algún caso de infección por S. multivorum en pacientes grandes quemados o con quemaduras extensas.

\section{Conclusión}

El rol patógeno del S. multivorum en humanos ha sido recientemente reconocido. Las infecciones por $S$. multivorum son usualmente de adquisición intrahospitalaria y en pacientes con alguna comorbilidad; sin embargo, también se ha descrito en individuos inmunocompetentes.

En pacientes quemados con signos de sepsis se deben tomar hemocultivos y cultivos de tejido de la zona de la quemadura a fin de documentar microorganismos, a veces inusuales, con el fin de adecuar el tratamiento antibacteriano y así reducir la morbimortalidad asociada. 


\section{Caso clínico}

\section{Referencias bibliográficas}

1.- Vella Ramírez J C, Simón Rodríguez A. Infección respiratoria por Sphingobacterium multivorum. An Med Interna (Madrid) 2001; 18: 655-6.

2.- Azarfar A, Bhattacharya S, Siddiqui S, Subhani N. Sphingobacterium thalpophilum bacteraemia: A case report. Int J Infect Dis 2020; 18: 1-4. doi: 10.5580/IJID.55030.

3.- Aydogan M, Yumuk Z, Dündar V, Arisoy E S. Sphingobacterium multivorum septicemia in an infant: report of a case and review of the literature. Türk Mikrobiyol Cemiy Derg 2006; 36: 44-8.

4.- Verma R K, Rawat R, Singh A, Singh D P, Verma V. Sphingobacterium multivorum causing fatal meningoencephalitis: a rare case report. Int J Res Med Sci 2014; 2: 1710-2. doi: 10.5455/2320-6012.ijrms20141189

5.- $\quad$ Steinberg J, Lutgring J, Burd E. Other Gram-Negative and Gram-Variable Bacilli. Mandell G, Bennett J, Dolin R, eds. Principles and Practice of Infectious Diseases, 9th ed. Philadelphia: Elsevier; 2019: p. 2847-62.

6.- Tronel H, Plesiat P, Ageron E, Grimont P
A D. Bacteremia caused by a novel species of Sphingobacterium. Clin Microbiol Infect 2003; 9: 1242-4. doi: 10.1111/j.14690691.2003.00801.x

7.- Barahona F, Slim J. Sphingobacterium multivorum: case report and literature review. New Microbes New Infect 2015; 7: 33-6. doi: 10.1016/j.nmni.2015.04.006.

8.- Freney J, Hansen W, Ploton C, Meugnier $\mathrm{H}$, Madier S, Bornstein N, et al. Septicemia caused by Sphingobacterium multivorum. J Clin Microbiol 1987; 25: 1126-8. doi: 10.1128/ JCM.25.6.1126-1128.1987.

9.- Abro A H, Rahimi Shahmirzadi M R, Jasim L M, Badreddine S, Al Deesi Z. Sphingobacterium multivorum bacteremia and acute meningitis in an immunocompetent adult patient: A case report. Iran Red Crescent Med J 2016; 18: e38750. doi: 10.5812/ ircmj.38750.

10.- Reina J, Borrell N, Figuerola J. Sphingobacterium multivorum isolated from a patient with cystic fibrosis. Eur J Clin Microbiol Infect Dis 1992; 11: 81-2. doi: 10.1007/BF01971283.

11.- Areekul S, Vongsthongsri U, Mookto
T, Chettanadee S, Wilairatana P. Sphingobacterium multivorum septicemia: a case report. J Med Assoc Thai 1996; 79: 395-8.

12.- Khalid M, Khan I, Hashmi A, Kanna M, Lewis P, Patel P. An interesting case of Sphingobacterium multivorum neck abscess. AJRID 2018; 1: 1-5. doi: 10.9734/ajrid/2018/ v1i113941.

13.- Abensur H, Dantas F, Araujo M, Campos R, Araujo M, Romao J. Infective endocarditis related to the uncommon gramnegative pathogen Sphingobacterium spiritivorum. Clin Microbiol Infect Dis 2019; 4: 1-3. doi: 10.15761/CMID. 1000160.

14.- Potvliege C, Dejaegher-Bauduin C, Hansen W, Dratwa M, Collart F, Tielemans C, et al. Flavobacterium multivorum septicemia in a hemodialyzed patient. J Clin Microbiol 1984; 19: 568-9. doi: 10.1128/JCM.19.4.568569.1984.

15.- Pernas-Pardavila H, Vallejo-Alonso AM, NovoVeleiro I, Navarro de la Cruz D, GonzálezQuintela A. Sphingobacterium multivorum: An atypical bacterium in an atypical place. Eur J Case Rep Intern Med 2019; 6: 001214. doi: 10.12890/2019 001214 . 